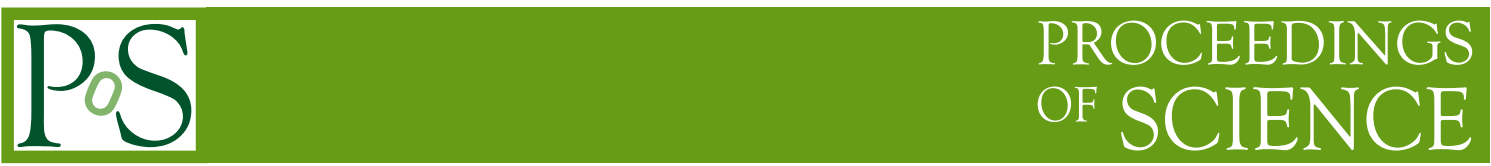

\title{
Neutrino Oscillation Studies in JUNO
}

\author{
Wenjie Wu*, on behalf of the JUNO collaboration \\ School of Physics and Technology, Wuhan University, Wuhan 430072, China \\ E-mail: wuwenjie@ihep.ac.cn
}

\begin{abstract}
Neutrino oscillation is the first observed phenomenon beyond the Standard Model, which can be interpreted in the framework of three neutrino mixings. It is governed by three mixing angles, two mass-squared differences, and one Dirac $\mathrm{CP}$ violation phase. The neutrino mass ordering, $\mathrm{CP}$ violation phase and the octant of $\theta_{23}$ remain unknown and could be measured by next-generation neutrino oscillation experiments. Jiangmen Underground Neutrino Observatory (JUNO) consists of a 20 kiloton liquid scintillator detector, which primarily aims to determine the neutrino mass ordering by measuring the reactor antineutrino oscillation pattern at a baseline of about $53 \mathrm{~km}$. A $3-4 \sigma$ significance to resolve mass orderings could be reached after 6 years of operation benefiting from its unprecedented 3\% energy resolution at $1 \mathrm{MeV}$. Besides, JUNO is able to perform measurements at an accuracy of better than $1 \%$ to oscillation parameters $\sin ^{2} \theta_{12}, \Delta m_{21}^{2}$ and $\Delta m_{32}^{2}$. In this talk, neutrino oscillation studies in JUNO will be reviewed.
\end{abstract}

The 21st international workshop on neutrinos from accelerators (NuFact2019)

August 26 - August 31, 2019

Daegu, Korea

${ }^{*}$ Speaker. 


\section{Introduction}

Neutrino oscillation originates from the mixture between the mass and flavor eigenstates of neutrinos, which can be described by the PMNS matrix. In the framework of three generations of neutrinos, six parameters are involved in neutrino oscillation. $\sin ^{2} \theta_{12}, \sin ^{2} \theta_{23}, \sin ^{2} \theta_{13}, \Delta m_{21}^{2}$ and $\left|\Delta m_{31}^{2}\right|$ have been measured at the precision of $4.5 \%, 5.2 \%, 3.4 \%, 2.4 \%$ and $2.6 \%[1,2,3,4,5]$. The sign of $\Delta m_{31}^{2}$, the octant of $\theta_{23}$ and the leptonic CP violation phase $\delta$ remain unknown. The sign of $\Delta m_{31}^{2}$ raises the question that whether $v_{3}$ is heavier (Normal ordering) or lighter (Inverted ordering) than $v_{2}$.

The Jiangmen Underground Neutrino Observatory (JUNO) is a multi-purpose reactor neutrino experiment [6]. It aims to determine the neutrino mass ordering and precisely measure the oscillation parameters. The central detector of JUNO contains 20 kiloton liquid scintillator (LS) within an acrylic sphere with inner radius of $17.7 \mathrm{~m}$. Double calorimeter system consists of 18,000 20inch PMTs and 25,000 3-inch PMTs which are mounted on the stainless steel latticed shell and immersed in the water buffer. Benefiting from the highly transparent LS and high photocathode coverage, JUNO can achieve an unprecedented energy resolution of $3 \% / \sqrt{E(\mathrm{MeV})}$.

\section{Measurement of neutrino mass ordering in JUNO}

In the framework of three generations of neutrinos, the survival probability of $\bar{v}_{e}$ in vacuum can be written as $[6,7]$

$$
\begin{aligned}
P_{\bar{v}_{\mathrm{e}} \rightarrow \bar{v}_{\mathrm{e}}} & =1-\sin ^{2} 2 \theta_{13}\left(\cos ^{2} \theta_{12} \sin ^{2} \Delta_{31}+\sin ^{2} \theta_{12} \sin ^{2} \Delta_{32}\right)-\cos ^{4} \theta_{13} \sin ^{2} 2 \theta_{12} \sin ^{2} \Delta_{21} \\
& =1-2 s_{13}^{2} c_{13}^{2}+2 s_{13}^{2} c_{13}^{2} \sqrt{1-4 s_{12}^{2} c_{12}^{2} \sin ^{2} \Delta_{21}} \cos \left(2\left|\Delta_{32}\right| \pm \phi_{e e}\right)-4 c_{13}^{4} s_{12}^{2} c_{12}^{2} \sin ^{2} \Delta_{21} \\
& =a_{0}+a_{1}(\xi) \cdot \cos \left[\left(2\left|\Delta m_{32}^{2}\right| \pm \Delta m_{\phi_{e e}}^{2}\right) \cdot \xi\right]-P_{\odot}(\xi)
\end{aligned}
$$

where

$$
\begin{aligned}
s_{i j} & =\sin \theta_{i j}, c_{i j}=\cos \theta_{i j}, \xi=\frac{L}{4 E}, \Delta_{i j}=\frac{\Delta m_{i j}^{2} L}{4 E}, \Delta m_{\phi_{e e}}^{2}=\frac{4 E \phi_{e e}}{L}, \\
\phi_{e e} & =\tan ^{-1}\left(\frac{\cos ^{2} \theta_{12} \sin 2 \Delta_{21}}{\cos ^{2} \theta_{12} \cos 2 \Delta_{21}+\sin ^{2} \theta_{12}}\right) .
\end{aligned}
$$

The \pm sign of Equation 2.1 is decided by the $\mathrm{MO}$ with plus sign for the normal $\mathrm{MO}$ and minus sign for the inverted MO. The interference of $\Delta m_{31}^{2}$ and $\Delta m_{32}^{2}$ gives a beat pattern to the fast oscillation term of survival probability, as shown in Figure 1. The extra effective mass-squared difference $\Delta m_{\phi_{e e}}^{2}$ is relevant to the distortion of the energy spectrum giving a specific baseline. Figure 2 shows $\Delta m_{\phi_{e e}}^{2}$ as a function of baseline and visible prompt energy $E_{\mathrm{vis}} \simeq E_{\mathrm{n}}-0.8 \mathrm{MeV}$ [7]. Therefore, the advancement or retardation of the oscillation phase contains useful MO information.

In the sensitivity study, the benchmark is assuming a $20 \mathrm{kt} \mathrm{LS}$ detector and the total thermal power of the two reactor complexes as $36 \mathrm{GW}_{\text {th }}$ with the nominal running time of 6 years, $73 \%$ efficiency and an energy resolution of $3 \% / \sqrt{E(\mathrm{MeV})}$. The $\chi^{2}$ test shows that a sensitivity of $\Delta \chi_{\mathrm{MO}}^{2} \simeq 16$ can be obtained in the ideal case as shown in Figure 3. The decomposition of systematics from reactor and backgrounds are shown in Table 1 . The sensitivity could be enhanced with 


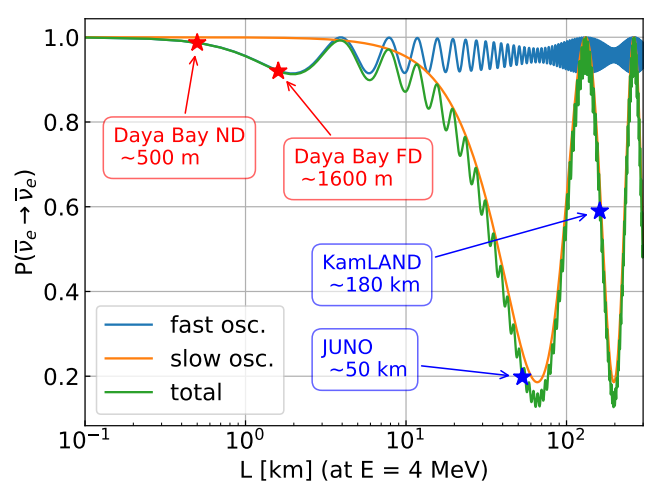

Figure 1: Survival probability of $\bar{v}_{e}$ with energy of $4 \mathrm{MeV}$.

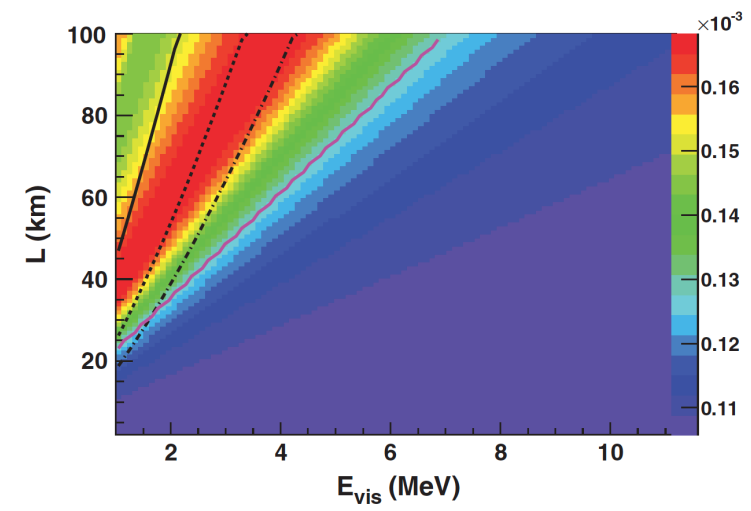

Figure 2: $\Delta m_{\phi_{e e}}^{2}$ as a function of baseline $L$ and visible prompt energy $E_{\mathrm{vis}}[7]$.

additional information from the measurement of $\left|\Delta m_{\mu \mu}^{2}\right|$ in long-baseline muon-neutrino oscillation experiments. The enhancement of $\Delta \chi_{\mathrm{MO}}^{2}$ ranges from 4 to 12 for the $1 \%$ relative precision of $\left|\Delta m_{\mu \mu}^{2}\right|$ considering the whole parameter space of the CP phase.

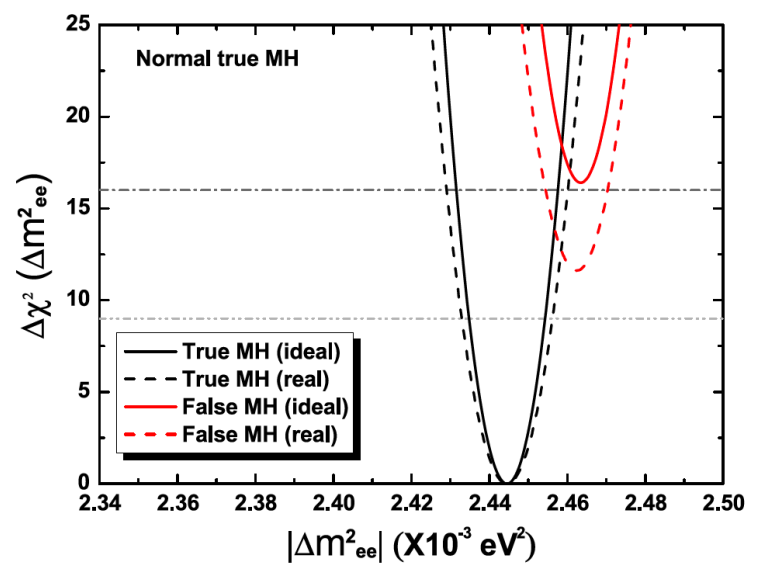

Figure 3: The comparison of the MO sensitivity for the ideal and real distribution of the reactor cores. The real distribution including DYB and HZ NPPs gives a degradation of $\Delta \chi_{\mathrm{MO}}^{2} \simeq 5$ [6].

\begin{tabular}{cccccccc}
\hline & Ideal & Core distr. & DYB and HZ & Reactor (shape) & B/S (rate) & B/S (shape) & $\left|\Delta m_{\mu \mu}^{2}\right|$ \\
\hline Size & $52.5 \mathrm{~km}$ & Real & Real & $1 \%$ & $6.3 \%$ & $0.4 \%$ & $1 \%$ \\
$\Delta \chi_{\mathrm{MO}}^{2}$ & 16 & -3 & -1.7 & -1 & -0.6 & -0.1 & $+(4-12)$ \\
\hline
\end{tabular}

Table 1: Different contributions to the MO sensitivity [6].

\section{Precision measurement in JUNO}

Besides the determination of the neutrino MO, JUNO is expected to give a precise measurement of three oscillation parameters, including $\sin ^{2} 2 \theta_{12}, \Delta m_{21}^{2}$ and $\Delta m_{32}^{2}\left(\Delta m_{31}^{2}\right)$. The precision measurement of neutrino oscillation parameters is a powerful tool to test the standard three-flavor 
neutrino model. Current precision of oscillation parameters from individual experiments and a global fit are shown in Table 2. With the nominal setup described in the MO sensitivity study, the expected accuracy for the three relevant parameters of JUNO are $0.24 \%, 0.27 \%$ and $0.54 \%$ respectively. After considering additional systematic uncertainties from rector neutrino spectrum, detector energy scale and backgrounds, the precision can reach $0.59 \%, 0.44 \%$ and $0.67 \%$ respectively. With such high precision of oscillation parameters, JUNO can help in probing the unitary of the PMNS matrix to the level of $1 \%$.

\begin{tabular}{cccccc}
\hline & $\Delta m_{21}^{2}$ & $\left|\Delta m_{31}^{2}\right|$ & $\sin ^{2} \theta_{12}$ & $\sin ^{2} \theta_{13}$ & $\sin ^{2} \theta_{23}$ \\
\hline Dominant Exps. & KamLAND & T2K & SNO+SK & Daya Bay & NOvA \\
Individual 1 $\sigma$ & $2.4 \%[4]$ & $2.6 \%[5]$ & $4.5 \%[1]$ & $3.4 \%[3]$ & $5.2 \%[2]$ \\
Nu-FIT 4.1 [8] & $2.7 \%$ & $1.3 \%$ & $4.2 \%$ & $2.9 \%$ & $3.6 \%$ \\
JUNO (statistics) & $0.24 \%$ & $0.27 \%$ & $0.54 \%$ & - & - \\
JUNO (w/ systematics) & $0.59 \%$ & $0.44 \%$ & $0.67 \%$ & - & - \\
\hline
\end{tabular}

Table 2: Precision for oscillation parameters from individual experiments and a global fit. And the expected precision for three relevant oscillation parameters of JUNO.

\section{Summary and Prospect}

JUNO could largely advance the neutrino physics with its unprecedented energy resolution and high statistics. The sensitivity study shows that a $3-4 \sigma$ significance to determined neutrino MO can be reached after six years of operation. In addition, JUNO can measure $\sin ^{2} 2 \theta_{12}, \Delta m_{21}^{2}$ and $\Delta m_{32}^{2}\left(\Delta m_{31}^{2}\right)$ to world-leading precisions. JUNO will be the first experiment to simultaneously observe an oscillation pattern containing two independent frequencies and more than two oscillation cycles.

\section{References}

[1] M. Ikeda, Solar neutrino measurements with super-kamiokande, June, 2018. http://doi.org/10.5281/zenodo.1286858.

[2] M. Sanchez, NOvA Results and Prospects, June, 2018. http://doi.org/10.5281/zenodo.1286758.

[3] The Daya Bay Collaboration, Measurement of the Electron Antineutrino Oscillation with 1958 Days of Operation at Daya Bay, Physical Review Letters 121 (2018) 241805.

[4] A. Suzuki, Antineutrino science in KamLAND, The European Physical Journal C 74 (2014) 3094.

[5] M. Wascko, T2K Status, Results, and Plans, June, 2018. http://doi.org/10.5281/zenodo.1286752.

[6] F. An, et al., Neutrino physics with JUNO, Journal of Physics G: Nuclear and Particle Physics $\mathbf{4 3}$ (2016) 030401.

[7] X. Qian, D. A. Dwyer, R. D. McKeown, P. Vogel, W. Wang and C. Zhang, Mass hierarchy resolution in reactor anti-neutrino experiments: Parameter degeneracies and detector energy response, Physical Review D 87 (2013) 033005.

[8] I. Esteban, M. C. Gonzalez-Garcia, A. Hernandez-Cabezudo, M. Maltoni and T. Schwetz, Global analysis of three-flavour neutrino oscillations: Synergies and tensions in the determination of $\theta_{23}$, $\delta_{\mathrm{cp}}$, and the mass ordering, Journal of High Energy Physics 2019 (2019) 106. 